\title{
Investigations of Coherent Vibrational Oscillations in Myoglobin
}

\author{
Florin Rosca, Anand T. N. Kumar, Xiong Ye, Theodore Sjodin, Andrey A. Demidov, and \\ Paul M. Champion*
}

Department of Physics and Center for Interdisciplinary Research on Complex Systems, Northeastern University, Boston, Massachusetts 02115

Received: October 11, 1999

\begin{abstract}
The technique of femtosecond coherence spectroscopy (FCS) is applied to the heme protein myoglobin. Photostable samples of deoxy myoglobin $(\mathrm{Mb})$ and photochemically active samples of the nitric oxide adduct (MbNO) are investigated. The pump-induced change in the probe transmittance for both samples displays coherent oscillations that, when transformed into the frequency domain, are in agreement with the resonance Raman spectrum of deoxy Mb. This indicates that the coherences associated with the photoreactive sample (MbNO) arise from the rapidly changing forces appearing in the crossing region(s) between the reactant and product state potential energy surfaces. The relative phase and amplitude of the Fe-His vibration, associated with the sole covalent linkage between the heme and the protein, are analyzed as a function of sample state and pump/probe carrier frequency. The dependence of the phase on carrier frequency is found to be significantly different for the "field driven" coherence in Mb and the "reaction driven" coherence in MbNO. In MbNO we observe a dip in amplitude and a phase flip near $439 \mathrm{~nm}$ for the $\mathrm{Fe}-$ His mode, whereas in deoxy $\mathrm{Mb}$ we observe a nearly constant phase and amplitude for this mode across the Soret absorption band. These observations are shown to be in good agreement with a simple theoretical model of the pump-probe experiment. Finally, we present recent observations of strong low-frequency oscillations, occurring near $40 \mathrm{~cm}^{-1}$ in both species and near $80 \mathrm{~cm}^{-1}$ for MbNO.
\end{abstract}

\section{Introduction}

Femtosecond coherence spectroscopy (FCS) is a pump-probe technique that utilizes ultrafast laser pulses to prepare and monitor coherent states in resonant or nonresonant samples. ${ }^{1-23}$ Some novel characteristics of FCS, compared with other standard spectroscopic techniques, include the capability of following ultrafast processes in real time so that the initial phases of vibrational oscillations can be measured and compared. For example, under certain conditions, FCS reveals the sign of the excited-state displacement, whereas resonance Raman spectroscopy is sensitive only to the absolute square of the excitedstate displacement. This technique can also follow coherences generated by rapid electronic state surface crossings that take place away from the vertical point of photon excitation. ${ }^{24}$ The spin selection rules associated with diatomic ligand photolysis in heme proteins lead to such nonvertical reactions, since the vertical (photon induced) electronic transition must preserve the spin of the ligand bound state $(S=0)$, which differs from that of the final dissociated product state $(S=2)$. Thus, the transition of the system from the $S=0$ reactant state to the $S=2$ product state must occur away from the vertical excitation point and is primarily dependent on the spin-orbit coupling. The ability to detect these "dark" reaction coupled coherences is a unique feature of FCS. ${ }^{11,15,25}$ Such coherences are significant because they provide a window to understanding the electronic and electron-nuclear coupling forces associated with the reaction coordinates of the "dark" chemical reaction.

In this paper we focus on the vibrational oscillations observed in myoglobin $(\mathrm{Mb})$ using FCS techniques. Myoglobin belongs to the large class of heme proteins, which are involved in a

* To whom the correspondence should be addressed. variety of physiologically important biochemical reactions such as electron transfer, diatomic molecule storage and transport, and catalysis. The heme group (active site) is a planar chromophore containing iron, which is bound to the "proximal" histidine (His) as the sole covalent link to the protein in $\mathrm{Mb}$. The heme iron binds diatomic molecules such as $\mathrm{NO}, \mathrm{CO}$, and $\mathrm{O}_{2}$, which can be photolyzed by a femtosecond laser pulse, rapidly creating a nonequilibrium structure. The relaxation dynamics of this nonequilibrium structure toward equilibrium has been extensively studied over broad time scales using a variety of techniques. ${ }^{26-43}$

In this work, we tune the laser carrier frequency into resonance with the strong Soret absorption band of myoglobin near $420-435 \mathrm{~nm}$ in order to both pump and probe the various sample states. This allows us to selectively probe the dynamics of the active site (heme) and its interaction with the protein. One of the observed nuclear vibrations, the $\mathrm{Fe}-\mathrm{His}$ mode, ${ }^{44}$ is found at $220 \mathrm{~cm}^{-1}$ and is an excellent indicator of the heme protein relative motions. The frequency, amplitude, and phase of the $\mathrm{Fe}-$ His mode observed in the FCS experiments are useful in helping us understand the dynamics of protein relaxation as well as the changes of the $\mathrm{Fe}-\mathrm{His}$ bond length between the various electronic states. The phase and amplitude measurements also allow us to directly differentiate between the field driven coherences in $\mathrm{Mb}$ and the reaction driven coherences in $\mathrm{MbNO}$.

Measurements as a function of detuning from the carrier frequency of the probe pulse (dispersed detection) are also presented to show how one can select for vibrational coherences based on the frequency content of the third-order polarization. ${ }^{25,45,46}$ The low-frequency modes that are revealed using the dispersed detection scheme are significant in that they likely represent important heme/protein motions that are intimately 
associated with the reaction coordinate for diatomic ligand binding. One motion that is believed to be important to the ligand binding and dissociation processes involves the "doming" of the heme group and the concomitant displacement of the iron atom from an in-plane position to roughly $0.4 \AA$ out of the heme plane. ${ }^{36,47,48}$ The low-frequency modes may also be connected to energy transport between the active site and the protein/ solvent interface. ${ }^{49-51}$ In this work, we present data that display strong oscillations near $40 \mathrm{~cm}^{-1}$ in both deoxy and NO bound myoglobin as well as the strong mode at $80 \mathrm{~cm}^{-1}$ reported earlier ${ }^{11}$ that is present only in the reaction driven samples (e.g., $\mathrm{MbNO}, \mathrm{MbCO}$ ). The presence of the $80 \mathrm{~cm}^{-1}$ mode in only those samples undergoing the photolysis reaction, where the iron displacements are known to be large, suggests that the 80 $\mathrm{cm}^{-1}$ mode is a strong candidate for the heme doming motion. ${ }^{11}$

\section{Materials and Methods}

We use a self-mode-locked Ti:sapphire laser (Coherent, Inc.) to generate the pump/probe pulses used in this work. The laser is capable of generating femtosecond pulses (45-80 fs) with a variable center (carrier) wavelength between 700 and $960 \mathrm{~nm}$. The pulses are generated with a repetition rate of $76 \mathrm{MHz}$ and are doubled using a $0.2 \mathrm{~mm} \mathrm{BBO}$ crystal so that the resulting blue pulses are used to both pump and probe the samples in the Soret absorption band. The average power of blue light at the sample is $30 \mathrm{~mW}$, which corresponds to about $0.5 \mathrm{~nJ}$ per pulse pair. The pump and probe beams are compressed using double pass prisms (SF10) for chirp compensation so that the time bandwidth product of the pulses is 0.46 , which is only $5 \%$ larger than the transform limit. The pump beam is modulated using an acousto-optic modulator (Neos Technologies) at approximately $1.5 \mathrm{MHz}$. The probe beam is passed through an optical delay line with a motorized translation stage that controls the pump-probe time delay. Both beams are focused into a rotating cell using a two inch lens in a near parallel geometry so that spatial filtration of the pump beam is possible. The polarizations of the pump and probe pulses are set to be perpendicular in order to improve the rejection of pump leakage into the detector. A lock-in amplifier (Stanford Research Systems SR844) is used to detect the small signal $\left(10^{-4}\right)$ induced on the probe intensity. Multiple scans of the optical delay line lead to the high signal/noise ratio necessary to detect the heme protein oscillations.

We use two kinds of detection: (1) a photodiode and (2) a monochromator-photomultiplier tube (PMT) combination. In the first case, the entire spectral bandwidth of the probe pulse is detected, which we refer to as an "open band" measurement. In the second case, the monochromator allows us to select a specific wavelength range, located within the probe pulse bandwidth, and we refer to such measurements as "dispersed" detection.

Horse heart myoglobin was purchased from Sigma Chemical Co. and used without further purification. The samples are prepared in a glovebox to prevent unwanted contamination from molecular oxygen. Potassium phosphate buffer $(\mathrm{pH}=7.8,0.1$ $\mathrm{M}$ ) is used to dissolve the protein, and $2 \mu \mathrm{L}$ of $1 \mathrm{M}$ dithionite solution is added to $90 \mu \mathrm{L}$ of buffered sample to obtain the deoxy $\mathrm{Mb}$ species. An additional $1 \mu \mathrm{L}$ of $1 \mathrm{M} \mathrm{NaNO}_{2}$ solution is added to prepare the $\mathrm{MbNO}$ adduct. The nitric oxide ligand is used in this study because it geminately rebinds to the heme iron in the 10-100 ps range, allowing for a complete reset of the sample prior to the arrival of the next pump-probe pulse pair.

The concentration of protein is chosen so that the sample has an absorbance of about 0.6 OD at the pump wavelength in a $0.5 \mathrm{~mm}$ cell. Each data set is the average of $60-120$ scans of the optical delay line, generated in a $2-4 \mathrm{~h}$ experiment. To ensure sample integrity, the absorption spectrum of the sample is measured before and after each experiment and intermediate FCS files are routinely compared.

Typical measured signals consist of damped oscillations imposed on a monotonically decaying background. To generate the power spectrum from the time-resolved data we use a linear predictive singular value decomposition (LPSVD) method that simultaneously fits both the oscillations and the monotonic decay. The plotted LPSVD power spectrum is analogous to the Fourier transform amplitude spectrum rather than the Fourier power spectrum, because it is linearly proportional to the oscillation amplitudes. An alternative method of analysis involves preliminary fitting of the monotonic background using the maximum entropy method (MEM), which generates a distribution of exponential decay rate constants. The residual oscillatory signal is subsequently fit using LPSVD or a Fourier transform. When the monotonic decay can be well described by exponential functions over the time range of interest (as is the case for the samples studied here), both techniques for fitting the monotonic background are found to generate the same power spectrum for the oscillatory signals.

\section{Experimental Results}

The femtosecond laser produces a pump pulse that can excite vibrational coherences lying within the pulse spectral bandwith ( $\left.~ 300-400 \mathrm{~cm}^{-1}\right)$, which are subsequently monitored by the probe pulse. When photostable samples (such as deoxy $\mathrm{Mb}$ ) are used, the coherences are driven by the pump electric fields and the forces associated with differences in the ground and resonant excited electronic state potential energy surfaces. In general, these field-driven coherences can be associated with vibrational states of both the ground and excited electronic state potential energy surfaces. However, in the case of myoglobin (and for heme proteins in general), the resonant $\pi-\pi^{*}$ Soret excited state is very short lived and population decay takes place within $10-100$ fs. ${ }^{52-57}$ Thus, the long-lived $(\sim 1-2$ ps) oscillatory signals that we observe are minimally complicated by optically driven vibrational coherences associated with the Soret excited electronic state.

The appearance of intermediate excited electronic states on the relaxation pathway remains a possibility, and there have been reports ${ }^{29,58,59}$ that indicate the existence of such states. However, Raman studies ${ }^{60,61}$ suggest that a majority of the population is directly channeled into the ground state vibrational manifold, while minor channels might lead to the appearance of small amounts of long-lived (ps time scale) intermediate states. ${ }^{61}$

A powerful method of studying the relaxation dynamics is the measurement of transient absorption spectra using a white light continuum. Figure 1a shows the evolution of the absorbance changes in the Soret region of $\mathrm{Mb}$ following Soret excitation $(400 \mathrm{~nm})$. The observed spectral features can be qualitatively interpreted as the cooling of a hot line shape that shifts to the blue and narrows as cooling takes place (note the early time evolution of the isosbestic point). Similarly, the spectral evolution of photolyzed MbNO, shown in Figure 1b, can be interpreted as the prompt appearance of the hot ground state of deoxy $\mathrm{Mb}$ followed by cooling. A quantitative analysis of the spectral changes introduced in Figure 1 will be presented elsewhere. ${ }^{62}$ The possibility of an excited state with a weak (or broadened) transition near $460 \mathrm{~nm}$ in deoxy $\mathrm{Mb}$ cannot be completely ruled out, but the main features of both the $\mathrm{Mb}$ and 
(a)
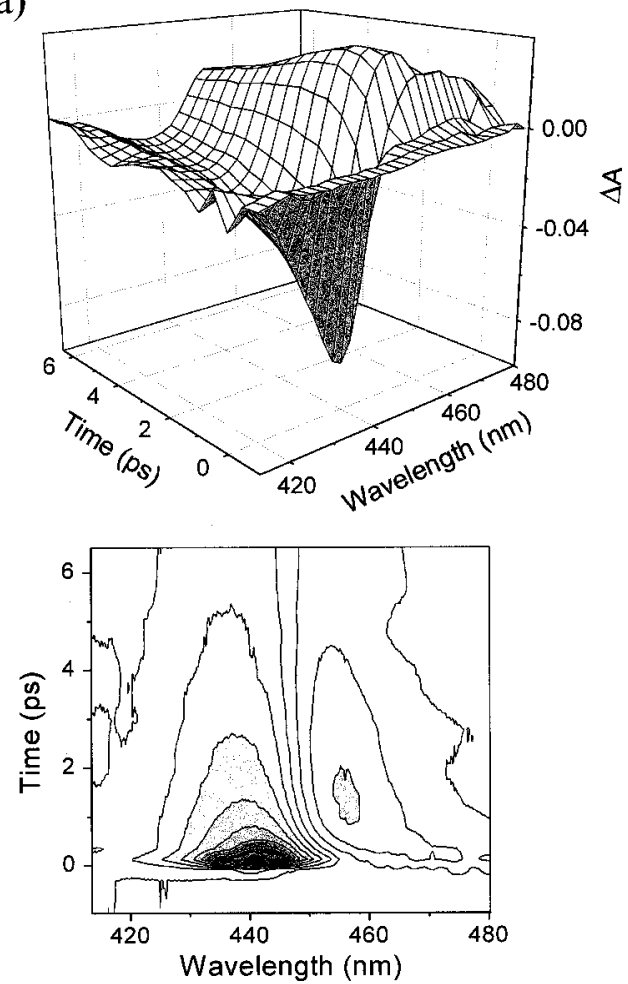

(b)
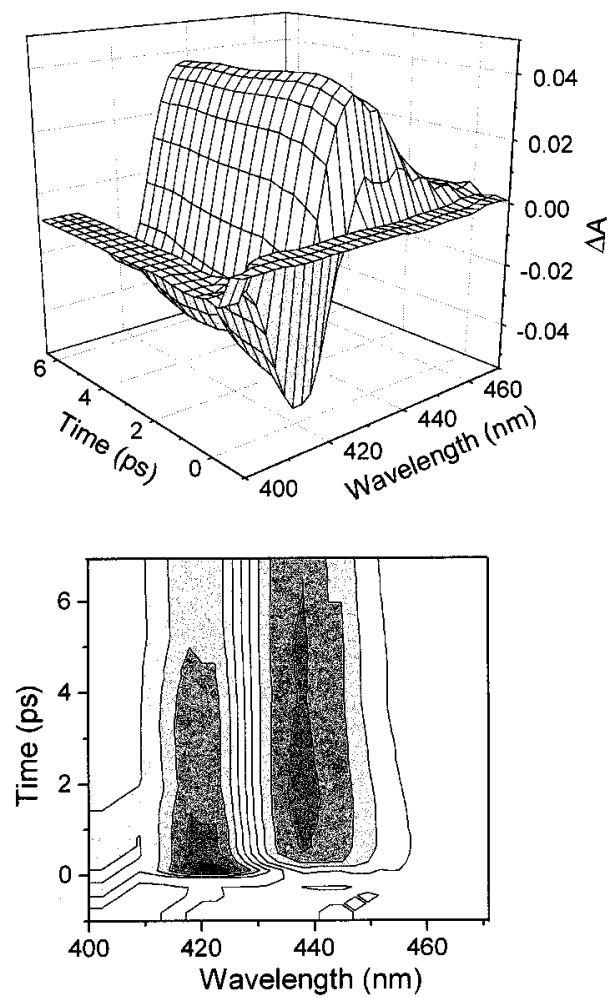

Figure 1. Transient absorption spectra displayed in 3D and contour plots: (a) deoxy Mb and (b) MbNO. Samples are excited by a 60 fs, $20 \mathrm{~nJ}$ laser pump pulse at $400 \mathrm{~nm}$.

MbNO spectral evolution are consistent with cooling of a hot heme. $^{42}$ Recent time-resolved Raman experiments on heme proteins demonstrate the immediate appearance of Raman spectra having peak positions within $5 \mathrm{~cm}^{-1}$ of the ground state peak positions with enhanced anti-Stokes intensities. ${ }^{60}$ These observations are also highly suggestive of the prompt formation of a vibrationally hot electronic ground state $\mathrm{Mb} .{ }^{60}$

Another strong argument for the prompt appearance of the electronic ground state of deoxy Mb comes from FCS, which probes the vibrational spectrum on still faster time scales. If the vibrational coherence is formed in the electronic ground state, the power spectrum of the observed FCS signals should resemble the resonance Raman spectrum of deoxy Mb. Figure $2 \mathrm{a}$ presents the FCS signals obtained from deoxy Mb in the dispersed detection scheme (open circles) along with the fit (solid line) using a linear predictive singular value decomposition (LPSVD) analysis. The signal consists of oscillations imposed on a monotonic decay. The monotonic decay is fit quite well with a double exponential, which is found to be selfconsistent with a maximum entropy analysis (Kumar et al., to be published) of the same data set. In Figure 2b we compare the LPSVD generated power spectrum from the fit of Figure $2 \mathrm{a}$ to the resonance Raman spectrum of deoxy $\mathrm{Mb}$. Because of the large detuning condition $(7 \mathrm{~nm})$, the low frequency and monotonic signals are strongly attenuated so that the higher frequency oscillations $\left(>100 \mathrm{~cm}^{-1}\right)$ can be compared with the resonance Raman spectrum. Notice that the fidelity between the FCS power spectrum and the Raman spectrum is quite good with the exception of the Raman peak at $304 \mathrm{~cm}^{-1}$, which is systematically shifted to $280-285 \mathrm{~cm}^{-1}$ in all samples investigated so far.

As previously mentioned, a unique feature of FCS is its ability to monitor the coherent dynamics triggered by a photon induced chemical reaction. The generated power spectra for a reaction driven process reveal the nuclear modes coupled to the reaction,
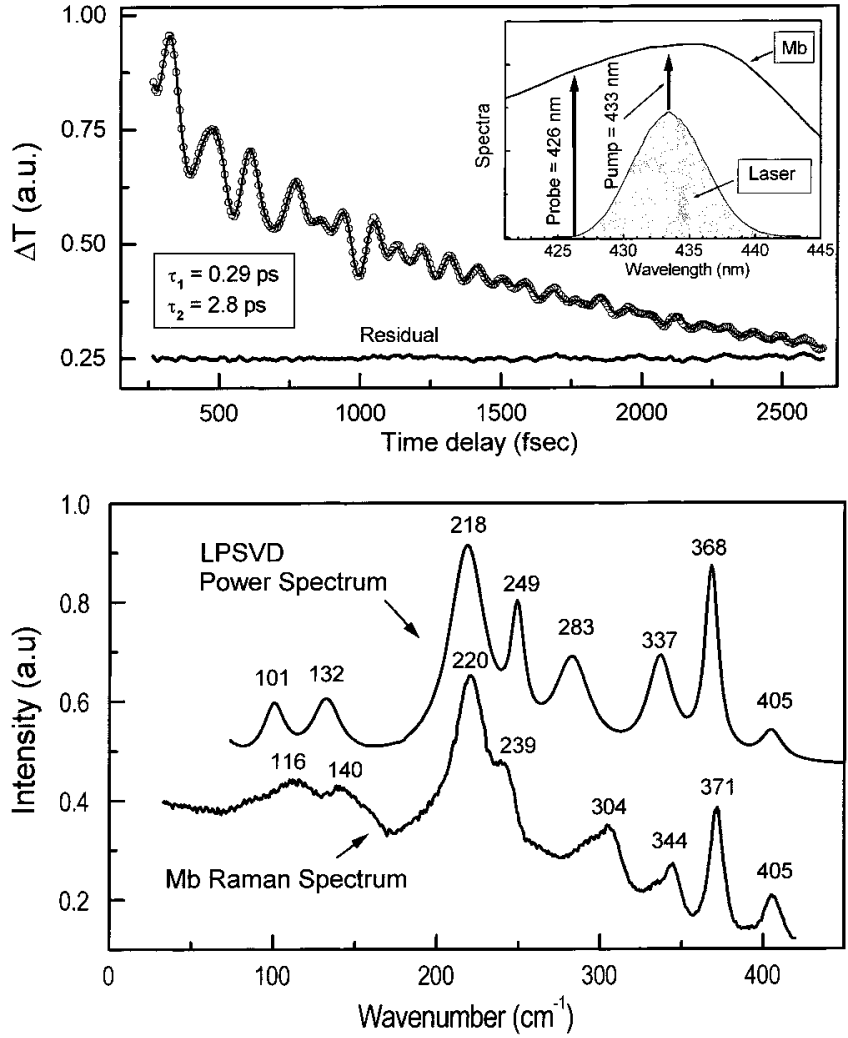

Figure 2. (upper panel) FCS signal from deoxy Mb. The circles are experimental data points and the solid line is the LPSVD fit. Two exponential time constants of 0.29 and 2.8 ps are found from the LPSVD fit, which characterize the monotonic background of the FCS signal. The inset shows the absorption spectrum of deoxy Mb, the spectrum of the laser pulse (grayed), and the detection wavelength. (lower panel) LPSVD generated power spectrum and the Raman spectrum of deoxy Mb. 

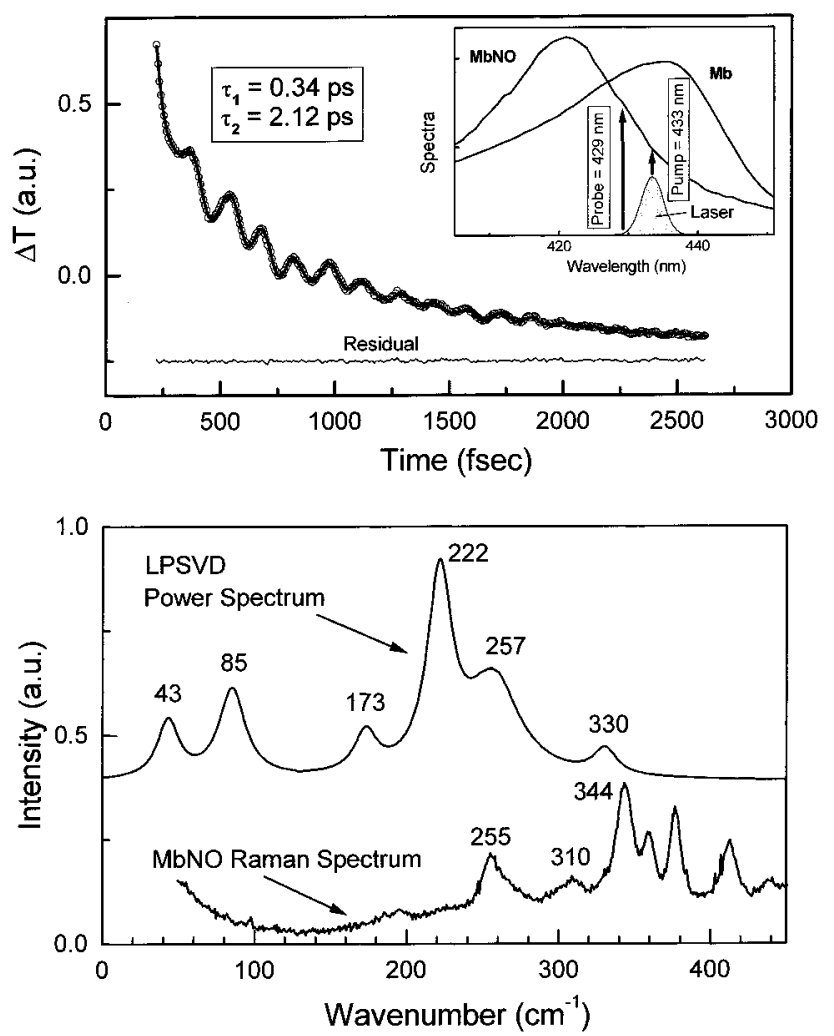

Figure 3. (upper panel) FCS signal from MbNO. The circles are experimental data points and the solid line is the LPSVD fit. Two exponential time constants 0.34 and 2.12 ps are found from the LPSVD fit, which characterize the monotonic background of the FCS signal. The inset shows equilibrium absorption spectra of MbNO (reactant) and the deoxy $\mathrm{Mb}$ (product), the spectrum of the laser pulse (grayed), and the detection wavelength. (lower panel) LPSVD generated power and the Raman spectrum of MbNO.

while the phase can be related to the direction of the reaction forces. As an example, we consider the NO photodissociation from $\mathrm{MbNO}$. The mechanism that drives the vibrational coherences in this case is essentially different ${ }^{24}$ from the one in deoxy $\mathrm{Mb}$ where the vibrational coherences are driven directly by the pump fields. Because of the different equilibrium structures of the reactant $(\mathrm{MbNO})$ and the product $(\mathrm{Mb})$, strong forces develop on the reaction coupled nuclei when the electronic surface crossings take place between the reactant $(S=0)$ and product $(S=2)$ states. Since the $\mathrm{Fe}-\mathrm{His}$ bond is approximately $0.1 \AA$ longer in the NO bound state of myoglobin compared to the deoxy state, ${ }^{63-65}$ the $\mathrm{Fe}-$ His mode is initially compressed and driven into oscillation by the NO dissociation. This generates a strong FCS signal for the $\mathrm{Fe}-\mathrm{His}$ mode with a uniquely determined phase.

Figure $3 \mathrm{a}$ displays the FCS signal from $\mathrm{MbNO}$ in the dispersed detection scheme (open circles) and the fit to the data (solid line) using LPSVD. In Figure $3 b$ we see that the LPSVD generated power spectrum contains the $\mathrm{Fe}-\mathrm{His}$ mode at 222 $\mathrm{cm}^{-1}$ (which is not active in the resonance Raman spectrum of $\mathrm{MbNO}$ ) and resembles the resonance Raman spectrum of the product state (deoxy Mb). Since less than $1 \%$ of the sample in the irradiated volume is photolyzed by the pump pulse, the observation of $\mathrm{Fe}-\mathrm{His}$ oscillations is strong evidence that most of the observed signal is generated by the electronic state changes that accompany NO dissociation. The observed signal has a linear dependence on the pump and probe intensities, excluding the presence of higher order nonlinear phenomena. The small peak near $250 \mathrm{~cm}^{-1}$ indicates the presence of some ground state field driven coherence associated with the MbNO reactant species. Nevertheless, the predominant signals in Figure 3 arise from those molecules in the sample that have absorbed a photon and dissociated NO, subsequently driving the modes comprising the reaction coordinate (e.g., Fe-His and heme doming) into coherent oscillation. ${ }^{24}$

As observed in cryogenic studies, there are Soret band spectral differences between myoglobin photolyzed at low temperature and unligated myoglobin in solution. ${ }^{34,66}$ This difference is characterized by a red shift in the Soret absorption band and an upshift of the $\mathrm{Fe}-\mathrm{His}$ mode frequency ${ }^{67}$ in the low temperature photoproduct. The FCS studies also reveal slightly different frequencies for the $\mathrm{Fe}-$ His mode in the case of deoxy $\mathrm{Mb}(219.8$ $\left.\pm 0.6 \mathrm{~cm}^{-1}\right)$ and $\mathrm{MbNO}\left(223.0 \pm 0.4 \mathrm{~cm}^{-1}\right)$ as determined using the statistics from 20 independent data sets. Experiments performed on samples prepared in a $75 \%$ glycerol solutions reveal an $\mathrm{Fe}-$ His frequency of $220 \mathrm{~cm}^{-1}$ for deoxy $\mathrm{Mb}$ (two independent data sets) and $225 \mathrm{~cm}^{-1}$ for MbNO (four independent data sets). The increased viscosity of glycerol slows down the conformational relaxation of the protein so that the $\mathrm{Fe}-\mathrm{His}$ mode is observed at higher frequency $\left(225 \mathrm{~cm}^{-1}\right)$ and evolves toward its equilibrium value of $220 \mathrm{~cm}^{-1}$. Moreover, the fact that we see a difference in the $\mathrm{Fe}-\mathrm{His}$ mode frequency for photolyzed MbNO between the aqueous $\left(223 \mathrm{~cm}^{-1}\right)$ and the $75 \%$ glycerol solutions $\left(225 \mathrm{~cm}^{-1}\right)$, demonstrates that the protein relaxation process in aqueous solution is taking place on a time scale of roughly 1 ps. This is consistent with earlier timeresolved Raman work showing that the relaxation of the $\mathrm{Fe}-$ His mode in $\mathrm{Mb}$ is completed within $30 \mathrm{ps}^{68}$

Since the detuning condition $(4 \mathrm{~nm})$ in Figure 3 is not so severe as in Figure $2(7 \mathrm{~nm})$, signals from lower frequency modes begin to appear and they can be identified at $43 \mathrm{~cm}^{-1}$ and $85 \mathrm{~cm}^{-1}$ in the power spectrum in Figure $3 \mathrm{~b}$. More detailed studies of these modes ${ }^{25}$ will be presented elsewhere; ${ }^{69}$ here we simply wish to stress the existence of the mode near 40 $\mathrm{cm}^{-1}$. Our earlier FCS studies ${ }^{11}$ clearly showed this mode in the power spectrum, but direct $40 \mathrm{~cm}^{-1}$ oscillations in the time domain data could not be discerned under the large detuning conditions that were employed in that study. As a result, we made no assignment of the $40 \mathrm{~cm}^{-1}$ feature. As will be shown below, the $40 \mathrm{~cm}^{-1}$ mode is definitely associated with the heme protein samples and robust oscillations with an $\sim 800 \mathrm{fs}$ period can be seen directly in both the detuning measurements and the open band data.

Figure 4 is an example of dispersed detection measurements, where the carrier frequency of the pump/probe pulse is positioned near the product state absorption maximum at 434 $\mathrm{nm}$. The detection monochromator, with band-pass of about 0.5 $\mathrm{nm}$, is systematically detuned from the carrier wavelength of the pump/probe pulse so that higher frequency vibrational components of the third order polarization are selected at larger detuning. This effect can be seen directly in the oscillatory components of the data in the left-hand panel of Figure 4. The right-hand panel shows the LPSVD power spectra found at each detuning wavelength. The main points to note here are that the $40 \mathrm{~cm}^{-1}$ mode is clearly a property of the sample and that the frequency content of the FCS signals can be selected using the dispersed detection scheme. ${ }^{25,45,46}$

In Figures 2 and 3, the probe pulse was dispersed through a monochromator, which was detuned from the carrier frequency, prior to detection by the PMT. When a detuning of several nm is employed, the FCS signals are filtered so that only the highfrequency vibrational components of the polarizability are observed. On the other hand, we have observed that when the detuning approaches $0 \mathrm{~nm}$ or when open band detection of the 

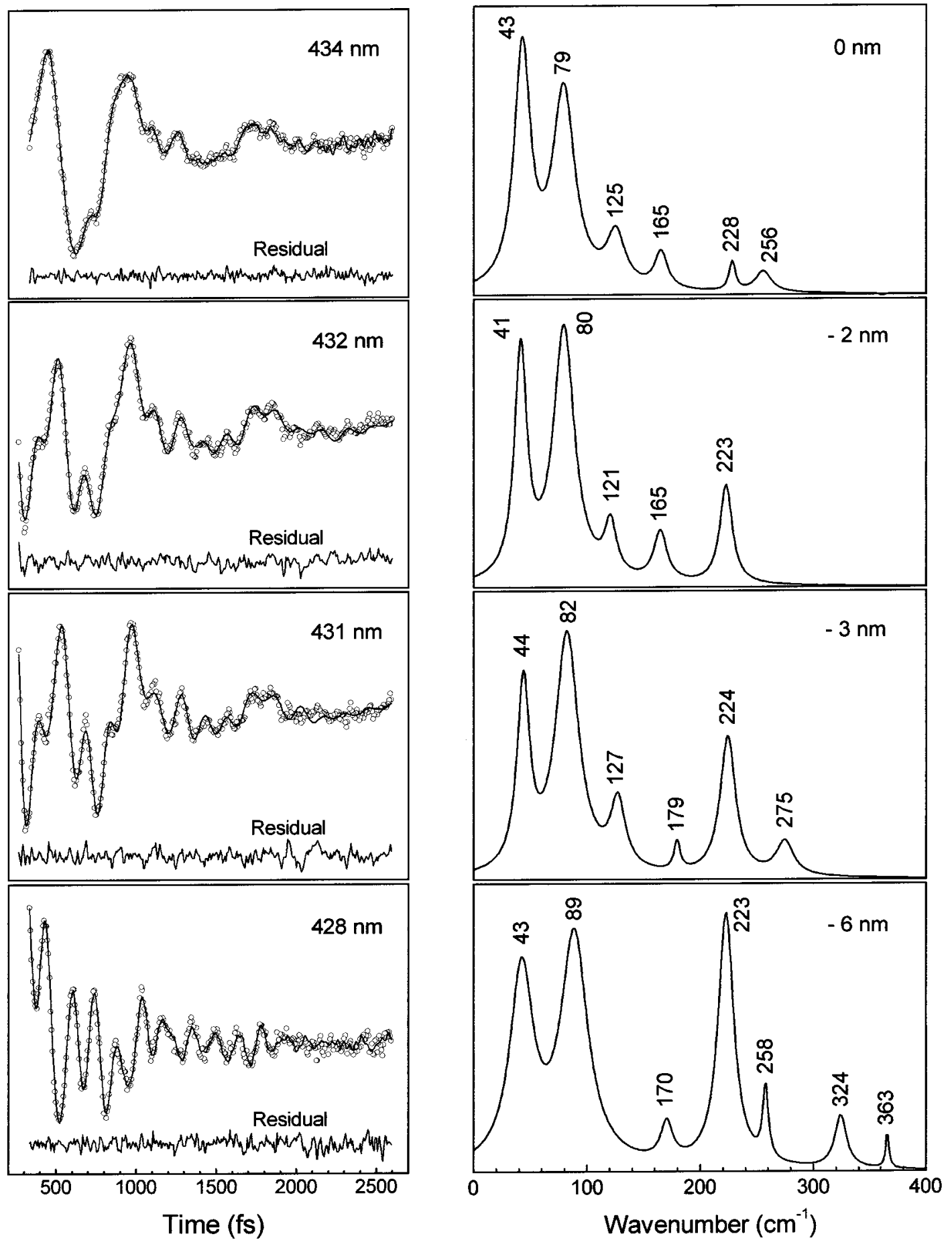

Figure 4. Sequence of experiments using dispersed detection. The MbNO sample is excited by $\sim 70 \mathrm{fs}$ pulses at $434 \mathrm{~nm}$. Detection is at 434 , 432 , 431, and $428 \mathrm{~nm}$. The left panels present the oscillatory part of the FCS signals (circles) with their LPSVD fits (solid line), whereas the right panels show the LPSVD generated power spectra.

probe pulse is used, it is possible to detect robust low-frequency modes. An important advantage of open band detection is that the autocorrelation measurement, used to determine the zero time delay between the pump and probe pulses, is also performed using this detection method. This allows the initial phases of the observed oscillations to be determined with improved accuracy compared to dispersed detection, since small frequency dependent time delays associated with probe pulse chirp can be eliminated.

We illustrate these observations in Figure 5, which displays the oscillatory part of the FCS signals for $\mathrm{Mb}$ and $\mathrm{MbNO}$ using open band detection at $427 \mathrm{~nm}$. For both samples a strong in- phase $40 \mathrm{~cm}^{-1}$ mode can be observed (thick solid line superimposed on the data). The observation of the same frequency in both samples suggests that both samples are driven into coherent motion on the same (presumably ground) electronic state surface. The components of the LPSVD fits representing the $220 \mathrm{~cm}^{-1}$ modes in the $\mathrm{Mb}$ and MbNO samples are displaced from the data and amplified by 4 to show the opposite phasing when the reaction and field-driven coherences are compared at $428 \mathrm{~nm}$. The vertical dashed lines are drawn to facilitate a direct visualization of the maxima and minima corresponding to the $220 \mathrm{~cm}^{-1}$ mode in the two data sets. The pump/probe carrier wavelength is chosen in this experiment to 


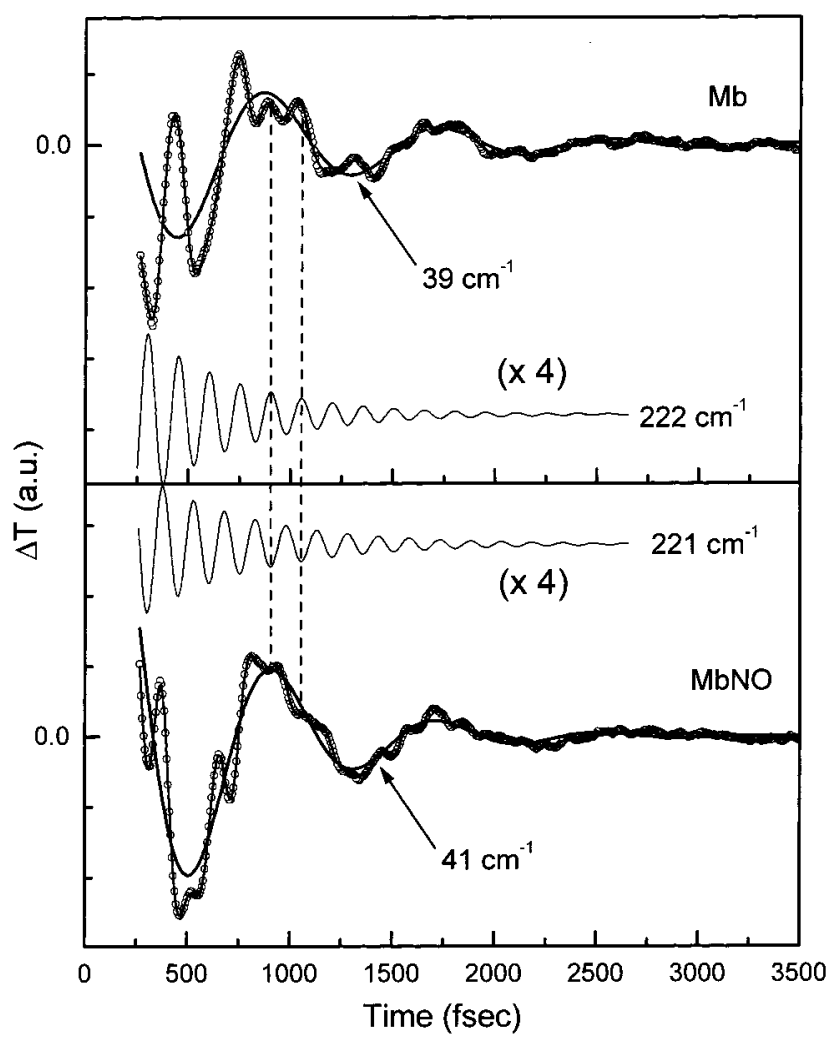

Figure 5. Oscillatory parts of the FCS signal (circles) in open band detection (pump and probe at $427 \mathrm{~nm}$, pulse duration $77 \mathrm{fs}$ ) from (a) deoxy $\mathrm{Mb}$ and (b) MbNO are displayed along with the LPSVD fits (solid lines). Superimposed are the $40 \mathrm{~cm}^{-1}$ (thick solid line) and 220 $\mathrm{cm}^{-1}$ (thin solid line) modes found in fitting procedure. For clarity the $220 \mathrm{~cm}^{-1}$ oscillations are vertically shifted and scaled up by a factor of 4 .

minimize the amplitude of the $80 \mathrm{~cm}^{-1}$ mode, so that it does not interfere with the $40 \mathrm{~cm}^{-1}$ mode in the MbNO sample.

We have also studied the behavior of the $\mathrm{Fe}-\mathrm{His}$ mode in deoxy and NO bound myoglobin over the 400-470 nm spectral range. Figure 6 shows a sequence of five open band measurements of MbNO near $439 \mathrm{~nm}$, where a dip in the intensity and an abrupt phase change of the Fe-His oscillatory signal are observed. The thin solid lines (displaced for clarity) show the $220 \mathrm{~cm}^{-1}$ component of the LPSVD fit, while the vertical dashed lines facilitate visualization of the $220 \mathrm{~cm}^{-1}$ phase change as a function of the carrier frequency. The heavy dashed lines show the $40 \mathrm{~cm}^{-1}$ LPSVD components. To minimize congestion in the figure, the LPSVD components associated with the $80 \mathrm{~cm}^{-1}$ mode are not shown. However, the contribution from this mode can be identified by the positive oscillations in the data near 400 and $800 \mathrm{fs}$.

The disappearance of the $220 \mathrm{~cm}^{-1}$ mode intensity at 439 $\mathrm{nm}$ and the accompanying phase change on either side of this wavelength are clearly discerned from the data presented in the left panel of the figure. Note that the phase change and loss of intensity of the $220 \mathrm{~cm}^{-1}$ mode at $439 \mathrm{~nm}$ is occurring near the absorption maximum of the product state (see Figure 1). This is further evidence that the measured coherence signals of the MbNO samples are arising from vibrational oscillations of the deoxy Mb product state.

In Figure 7, we present more detailed measurements of the phase of the $220 \mathrm{~cm}^{-1}$ mode in the MbNO sample as the pump/ probe carrier frequency is tuned over a broader spectral range. In the lower part of the figure, we show a schematic representation of the relevant energy surfaces describing the process. The potential well of the photoexcited reactant (MbNO*) along with the ground $(\mathrm{Mb})$ and Soret excited $\left(\mathrm{Mb}^{*}\right)$ potentials of the product state are shown as a function of the $\mathrm{Fe}-\mathrm{His}$ coordinate (Q). The dashed lines indicate pump/probe carrier frequencies that are detuned to the red and blue of the absorption maximum.

Following the spin-allowed Soret excitation to MbNO* by the pump pulse, the $\mathrm{Fe}-\mathrm{NO}$ bond lengthens (on a time scale that is roughly one-half the $\mathrm{Fe}-\mathrm{NO}$ vibrational period, $\sim 30$ fs) and spin state crossings take place leading to a deoxy $\mathrm{Mb}$ product state where the $\mathrm{Fe}-\mathrm{His}$ mode is positively displaced from equilibrium. The sign of this displacement follows from the fact that the $\mathrm{Fe}-\mathrm{His}$ bond is weakened and lengthened in the equilibrium $\mathrm{MbNO}$ state compared to the equilibrium deoxy $\mathrm{Mb}$ state. Note that $\mathrm{MbNO}$ and $\mathrm{MbNO}^{*}$ have the same $\mathrm{Fe}-$ His bond length, since the $\mathrm{Fe}-$ His mode is undisplaced and Raman inactive in MbNO upon Soret excitation. In the case of the photolysis reaction, the initial displacement of the product state vibrational wave packet for the $\mathrm{Fe}$-His mode is independent of the carrier frequency of the pump pulse. Thus, assuming ${ }^{70}$ that $\mathrm{Fe}-\mathrm{His}$ bond contraction takes place upon Soret excitation to $\mathrm{Mb}^{*}$, the probe pulse sees an initial antibleach (less transmission) when it is detuned to the blue of the absorption maximum. This leads to a phase of $\pi$ for the transmission signal, which is described by the function $A \cos (\omega t$ $+\varphi$ ). In contrast, when the carrier frequency of the probe is tuned to the red side of the absorption band, the probe pulse sees an initial bleach (more transmission), leading to a phase of zero for the transmission signal. This behavior is seen in the experimental data presented in the upper panel, which also shows the theoretical prediction (solid line) based on a fully quantum mechanical calculation in the well-separated pulse limit. ${ }^{71} \mathrm{We}$ emphasize that if the $\mathrm{Fe}-\mathrm{His}$ bond were expanded rather than contracted upon Soret excitation to $\mathrm{Mb}^{*}$ the absolute phases of the transmission signals would be reversed. This demonstrates how the phases of the FCS signals can be used to determine the absolute sign of excited state displacements and confirms Stavrov's prediction ${ }^{70}$ that the $\mathrm{Fe}-\mathrm{His}$ bond contracts upon Soret excitation.

In contrast to the distinct intensity and phase changes seen for the $\mathrm{Fe}-\mathrm{His}$ mode in the photoreactive MbNO sample, the open band oscillatory signals for the nonreactive (field driven) deoxy Mb sample show a nearly constant phase and the lack of a distinct dip in the intensity, for the $220 \mathrm{~cm}^{-1}$ mode. In Figure 8 we present open band measurements on deoxy $\mathrm{Mb}$ in the Soret region, and the corresponding phases of the $220 \mathrm{~cm}^{-1}$ mode are plotted in Figure 9a. It is important to note that the initial ground state wave packet displacement of a field-driven coherence depends critically on the detuning of the pump pulse carrier frequency relative to the center of the resonant absorption band. Figure $9 \mathrm{~b}$ schematically depicts the creation and detection of wave packet motion along the $\mathrm{Fe}-\mathrm{His}$ coordinate, for a degenerate pump-probe experiment, assuming a two electronic level system. A resonant pump pulse induces wave packet motion in the ground state with a momentum impulse (shown in the figure as an arrow above the wave packet) in the direction of increasing difference potential. ${ }^{72,73}$ Furthermore, when the carrier frequency is detuned toward the blue side of the resonant absorption maximum (corresponding to $\lambda_{\mathrm{B}}$ in Figure $9 \mathrm{~b}$ ), the wave packet is displaced toward decreasing bond lengths. This leads to an initially increased probe transmission, and the resulting phase is close to zero. On the other hand, as the pump carrier frequency is tuned to the red of the absorption maximum $\left(\lambda_{R}\right.$ in Figure $9 b$ ), the wave packet is displaced initially toward increasing bond lengths, and hence the phase of pump-probe 

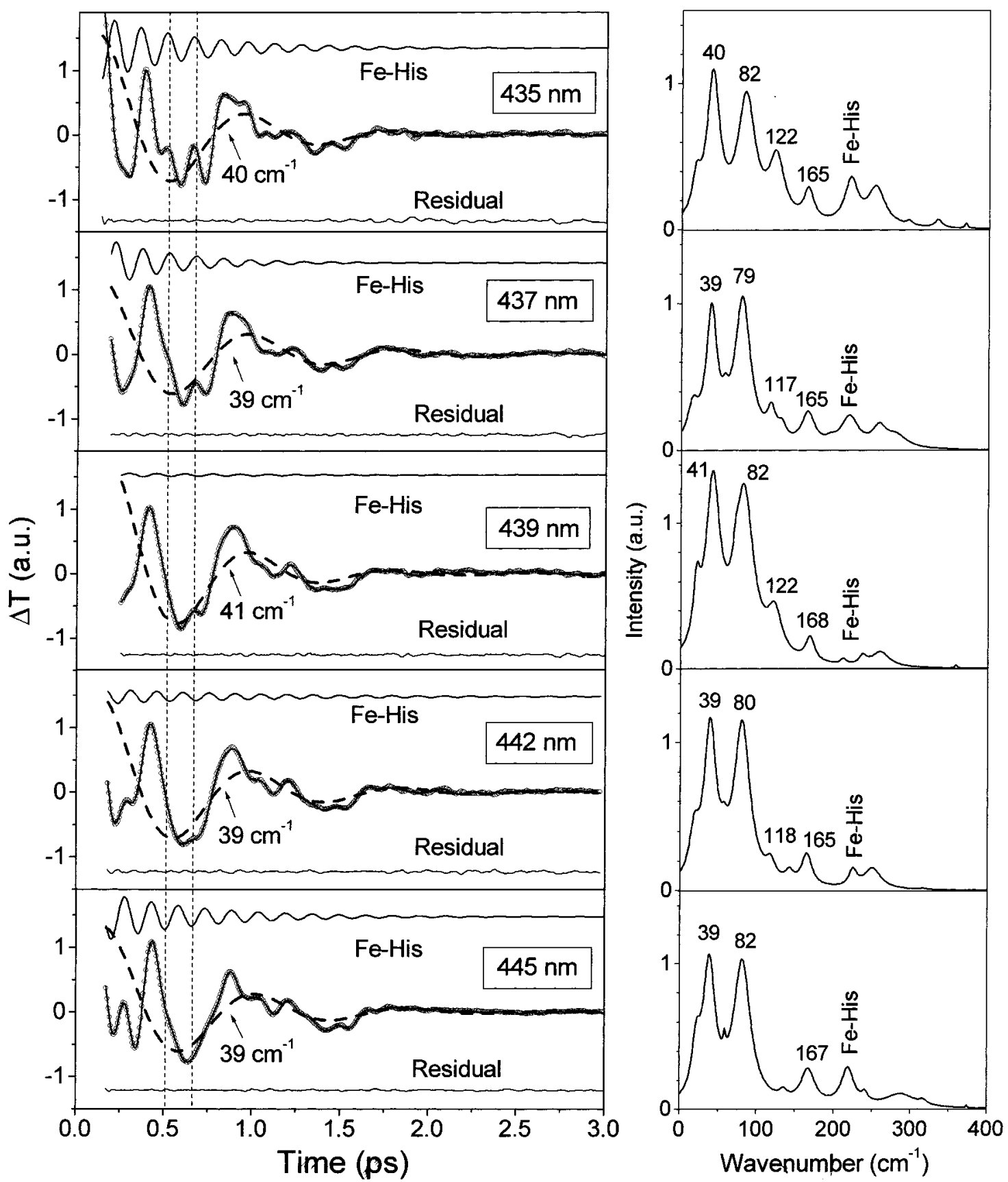

Figure 6. Sequence of open band FCS measurements on MbNO samples. The left panels present the oscillatory parts of the FCS signals (circles) with their LPSVD fits (solid line). The $40 \mathrm{~cm}^{-1}$ mode component (dashed line) is superimposed and the $220 \mathrm{~cm}^{-1}$ mode is shifted for clarity (thin solid line). The relative signal intensities at the different wavelengths are calibrated using the white light continuum measurements (Figure 1). Excitation and detection utilized 70-80 fs pulses at 435, 437, 439, 442, and $445 \mathrm{~nm}$. The right panels show the LPSVD-generated power spectra.

signal for red detuning is still expected to be near zero. (The expected results for a degenerate pump-probe experiment should be contrasted with the results one finds for a two-color experiment, ${ }^{8,9}$ where a fixed pump carrier frequency sets up an initial wave packet that is independent of probe carrier frequency, and results similar to Figure 7, with a distinct phase change near band center, are observed.)

The presence of momentum transfer from the pump pulse contributes to deviations of the predicted phase from zero. A fully quantum mechanical treatment of the pump pulse preparation step shows that the relative magnitudes of the momentum transfer and the displacement imparted to the wave packet (which determine its initial phase) depend on the temperature as well as the pump pulse carrier frequency. ${ }^{71}$ It is found that at high temperature $\left(h \omega \ll k_{\mathrm{B}} T\right)$ the induced wave packet displacement dominates the momentum transfer. However, in the region close to band center, the displacement approaches zero while the momentum is nonvanishing. This gives rise to deviations of the phase from zero near the center of the resonant region, where it undergoes a sharp and discontinuous jump between $\pm \pi / 2$ on either side of $\lambda_{\max }$. In Figure $9 \mathrm{a}$, we illustrate the predictions for the measured phase using a fully quantum mechanical theory of the pump-probe experiment in the wellseparated pulse limit. Our approach incorporates the measured equilibrium absorption line shape and the Kramers-Kronig determined dispersion line shape. The dashed line is obtained when the asymmetric experimental line shape of deoxy $\mathrm{Mb}$ at $T=300 \mathrm{~K}$ is used, while the solid line is the result using a Gaussian approximation. It can be seen that the approach to the off-resonant impulsive limit (when the phase approaches 
a)

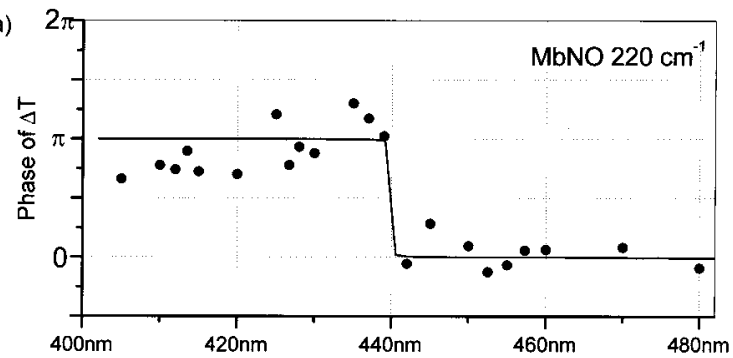

b)

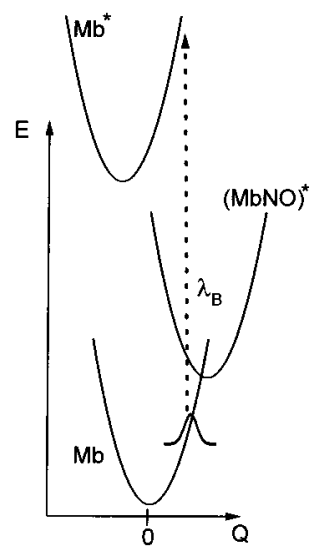

Reaction driven coherence

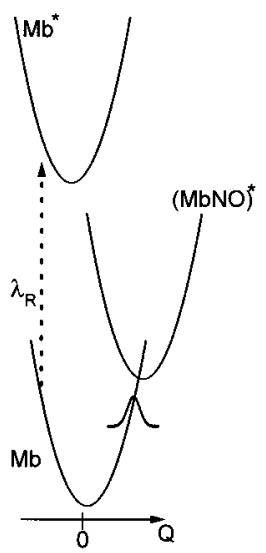

Figure 7. (a) Dependence of the phase of the $220 \mathrm{~cm}^{-1}$ mode of MbNO as the pump/probe carrier wavelength is varied. The circles are experimental data, and the solid line is the prediction based on a simple theoretical model for reaction-driven coherence in MbNO. The uncertainty in the phase estimation is about $\pm \pi / 4$. (b) The potential energy surfaces and the reaction wave packets along the $\mathrm{Fe}-\mathrm{His}$ coordinate $(\mathrm{Q})$ of the product state $(\mathrm{Mb})$. The dashed arrows indicate the probe carrier wavelength and $\mathrm{Mb}^{*}$ denotes the Soret excited state of the product. The pump pulse creates the reactant excited state (indicated as $\mathrm{MbNO}^{*}$ ), which rapidly decays to $\mathrm{Mb}+\mathrm{NO}$ along the transverse coordinate $R_{\mathrm{Fe}-\mathrm{NO}}$ and is not shown explicitly in the figure.

$\pi / 2$ ) occurs much more rapidly for the asymmetric line shape, due to the sharp decrease of the deoxy Mb absorption band at the red edge of the transition.

As can be seen from Figure 9, the predicted phase of the $220 \mathrm{~cm}^{-1}$ mode in deoxy $\mathrm{Mb}$ is in qualitative agreement with the experiment, except near the resonant region where the expected discontinuity near $435 \mathrm{~nm}$ is not clearly observed. As mentioned above, the approach of the theoretical phase to $\pm \pi / 2$ near the absorption maximum arises from the presence of nonzero momentum transfer between the pump pulse and the material. The center pair of potential surfaces in Figure 9b depicts the situation when the pump-probe carrier frequency is aligned with the absorption maximum. Under this condition, the wave packet merely receives a momentum impulse without a coordinate displacement, and we expect the intensity of the fundamental oscillations to vanish (the overtone intensity is a maximum under this condition). However, no clear dip in the intensity of the $220 \mathrm{~cm}^{-1}$ mode is observed experimentally, as seen in Figure 8. One possible reason for the deviation between the theoretical prediction and the experiment may be a nonoscillatory component of the deoxy $\mathrm{Mb}$ line shape that varies in time. From the dynamic absorption spectra presented in Figure $1 \mathrm{a}$, it is clear that the absorption line shape of photoexcited deoxy $\mathrm{Mb}$ is dynamic on the fs-ps time scale, especially in the wavelength region $441-435 \mathrm{~nm}$. This could potentially remove the dip in intensity of the $220 \mathrm{~cm}^{-1}$ mode, since the presence of a dip requires that the oscillatory dynamics of the line shape occur about a fixed absorption peak.

Thus, when the line shape exhibits a time dependent shift, we can expect that there is no fixed probe frequency for which oscillations are completely extinguished. It also turns out that the observed signal cannot be described by a simple cosine function so that the phase is ill-defined when probing within the dynamic region of the absorption band. Thus, the various fitting procedures, which assume simple cosine oscillations, return phases that are "averaged" over the shifting line shape and do not display evidence of the predicted $\pm \pi / 2$ phase discontinuity near band center. Another signature of the dynamic line shape model is an apparent increase in the damping of the vibrational oscillations, which arises as the line shape sweeps past the probe pulse carrier frequency. There is some evidence of this behavior in the $439 \mathrm{~nm}$ data shown in Figure 8 .

\section{Discussion}

The technique of FCS can play a major role in helping us to understand the nuclear motions that are involved in biomolecular reaction dynamics. We have shown how a nonreactive sample such as deoxy $\mathrm{Mb}$ can be driven into coherent motion by the action of the pump pulse fields and how these motions differ in phase and content from the coherent motions that are induced by a chemical reaction such as NO photolysis. In both cases, the FCS signals generate power spectra that are very similar to the resonance Raman spectrum of the deoxy sample. In particular, the $220 \mathrm{~cm}^{-1}$ mode, associated with the vibration of the $\mathrm{Fe}-$ His bond, shows phase behavior that demonstrates a contraction of the $\mathrm{Fe}$-His bond upon Soret excitation of deoxy $\mathrm{Mb}$. This experimental result is consistent with earlier predictions of the sign of the excited state potential energy surface displacements $^{70}$ and shows how FCS (in contrast to resonance Raman spectroscopy) can be utilized to obtain the sign of excited state displacements.

Moreover, the observed changes in the phase of the $220 \mathrm{~cm}^{-1}$ mode, as the pump/probe carrier frequency is tuned across the resonant absorption band, dramatically differentiates between field-driven and reaction-driven excitation of the coherent motion. The reaction-driven coherence shows a clear phase shift of $\pi$ as the carrier frequency moves through the center region of the absorption band. This is expected for a photolysis reaction that takes place independently of the pump carrier frequency. In contrast, the deoxy $\mathrm{Mb}$ sample displays no apparent phase shift, reflecting the fact that the pump pulse sets up an initial wave packet that depends on the pump carrier frequency. Thus, the results presented here confirm the idea ${ }^{11}$ that electronic forces, arising from the surface crossings associated with the $\mathrm{MbNO} \rightarrow \mathrm{Mb}+\mathrm{NO}$ chemical reaction, are the prime source of the coherent nuclear motions observed in the MbNO samples.

The theoretical predictions for a ground state field-driven coherence presented in Figure 9 are somewhat clouded by the lack of a clear dip in the $220 \mathrm{~cm}^{-1}$ mode intensity as the carrier frequency is tuned across the deoxy $\mathrm{Mb}$ absorption band. In addition, the observed phases, while in qualitative agreement with the theoretical predictions, do not show the expected $\pm \pi / 2$ dicontinuity as the carrier frequency approaches the band center region (435-445 $\mathrm{nm}$ ). One possible reason for this discrepancy is the assumption that the nonoscillatory component of the resonant absorption line shape has a time independent first moment. However, the presence of overdamped modes and/or anharmonicity invalidates this assumption and leads to monotonic time evolution in the first moment. This significantly affects the signals when the carrier wavelength of the probe pulse is in the vicinity of the peak of the shifting line shape. As can be clearly seen from the time dependent "isosbestic" 

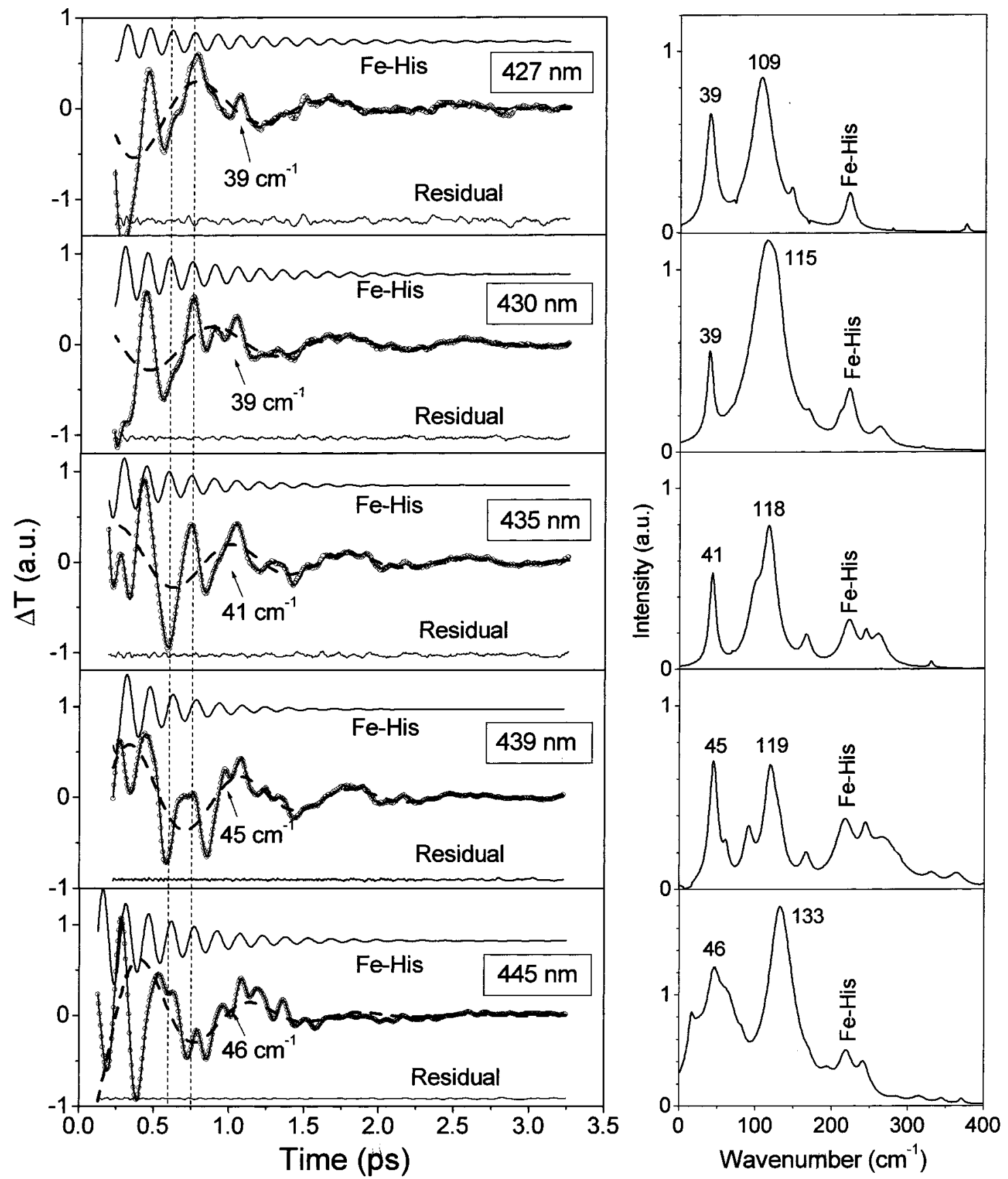

Figure 8. Sequence of open band measurements on deoxy Mb. The left panels present the oscillatory parts of the FCS signals (circles) with their LPSVD fits (solid line). The $40 \mathrm{~cm}^{-1}$ mode is superimposed (dashed line) and the $220 \mathrm{~cm}^{-1}$ mode is shifted for clarity (thin solid line). The wavelength dependence of the signal intensities is calibrated using the white light continuum experiment (Figure 1). Excitation and detection utilized 70-80 fs pulses at 427, 430, 435, 439, and $445 \mathrm{~nm}$. The right panels show the LPSVD generated power spectra.

point in Figure 1a, the probed absorption band in deoxy Mb is shifting during the first few picoseconds, so that the band center wavelength is changing. (Note that the transient absorbance of the MbNO photoproduct, Figure 1b, displays a much faster relaxation of the isosbestic point.) As mentioned above, the ps evolution of "band center" in the deoxy Mb sample can cause a blurring of the region over which a dip in the $220 \mathrm{~cm}^{-1}$ mode intensity would be seen and also affect the observed phases. A more formal theoretical treatment of these effects will be given elsewhere. ${ }^{71}$ Another theoretical issue that needs further consideration is the possibility that vibrational coherence created in the short-lived Soret excited state might contribute to the signal after nonradiative internal conversion to other longer lived states (including the ground state). The possibility that ultrafast nonradiative internal conversion processes can drive vibrational coherence (analogous to the reaction driven coherence) also needs to be explored. For simplicity, such effects have been ignored in the present discussion.

In addition to the quantitative phase and intensity measurements of the $220 \mathrm{~cm}^{-1}$ mode, we have also presented data to document the observation of a strong mode near $40 \mathrm{~cm}^{-1}$ in both the deoxy and $\mathrm{NO}$ bound myoglobin samples. The presence of this mode in deoxy $\mathrm{Mb}$ as well as in the NO bound sample (and all other heme samples studied to date) suggests that it is not driven by the dissociation reaction, but rather it may be a low-frequency mode, common to all heme protein samples. The universal appearance of the $\sim 40 \mathrm{~cm}^{-1}$ mode in the FCS signals of all heme proteins so far studied indicates that its observation in the cytochrome oxidase system is not unique, and therefore 

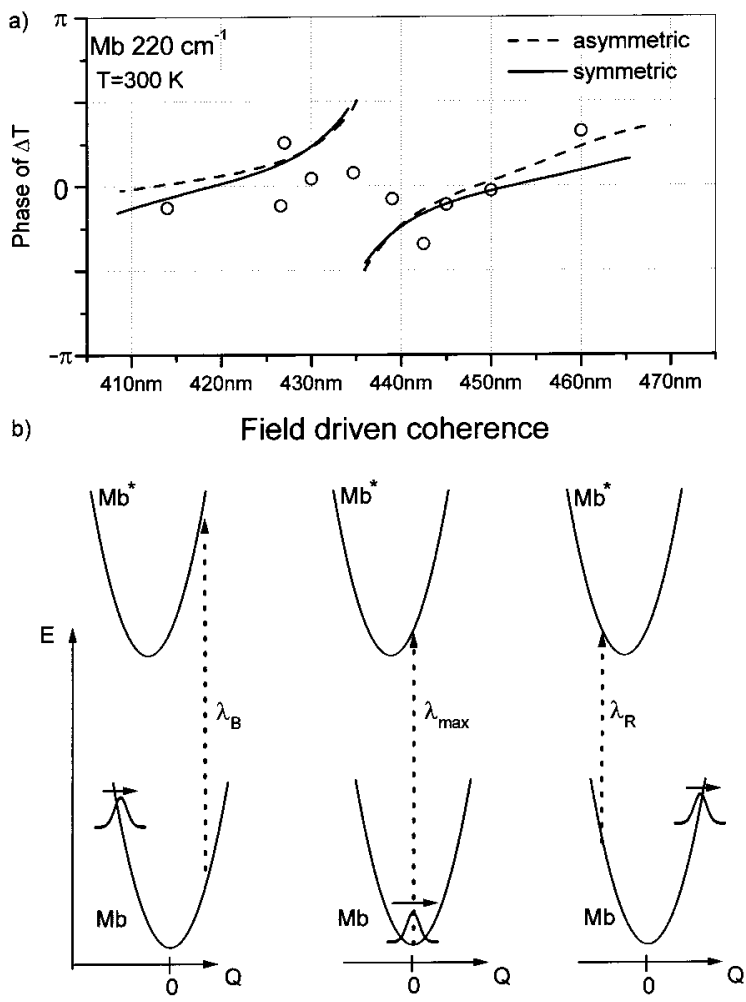

Figure 9. (a) Dependence of the phase of the $220 \mathrm{~cm}^{-1}$ mode in deoxy $\mathrm{Mb}$ on the pump/probe carrier wavelength. The experimental data are shown as circles and the solid line depicts the theoretical prediction for a $T=300 \mathrm{~K}$ symmetric Gaussian absorption line shape. The dashed line depicts the theoretical prediction for the measured asymmetric line shape of deoxy $\mathrm{Mb}$. The uncertainty in the phase estimation is about $\pm \pi / 4$. (b) The potential surfaces along the $\mathrm{Fe}-$ His coordinate for deoxy $\mathrm{Mb}$, shown with the ground-state wave packet created by the pump pulse. The dashed arrows indicate the pump-probe carrier wavelengths and the solid arrows above the wave packet indicate the direction and magnitude of the momentum imparted to the wave packet.

it is unlikely to be related to coherent population transfer as recently suggested. ${ }^{74}$

In contrast, a strong $80 \mathrm{~cm}^{-1}$ mode is seen exclusively in samples that undergo the ligand photolysis reaction, suggesting that it is the likely candidate for heme doming motion. Independent calculations of the doming frequency ${ }^{75,76}$ are supportive of this assignment. The possibility that the $80 \mathrm{~cm}^{-1}$ mode is an overtone of the $40 \mathrm{~cm}^{-1}$ mode, enhanced by the large difference in heme geometry between the reactant and product states (i.e., the $\sim 0.4 \AA$ displacement of $\mathrm{Fe}$ from the heme plane), has also been suggested. ${ }^{25} \mathrm{~A}$ third possibility is that a fundamental mode exists at $20 \mathrm{~cm}^{-1}$ (note the weak feature near $20 \mathrm{~cm}^{-1}$ in the power spectra of Figure 6) and that strong quadratic coupling enhances the even harmonics at 40 and $80 \mathrm{~cm}^{-1}$. However, quantitative theoretical calculations, using realistic values for the linear and quadratic coupling, suggest that the overtone and higher harmonic assignments are unlikely. ${ }^{71}$

Finally, we note that new high-resolution synchrotron based measurements, using the 14.36 Kev Mössbauer transition of Fe, have revealed the vibrational density of states that couple to the iron atom in $\mathrm{Mb}$ and MbCO. ${ }^{77}$ These low-temperature experiments find significant coupling to iron motion near 20 $\mathrm{cm}^{-1}$ and suggest that, if either the 40 or $80 \mathrm{~cm}^{-1}$ modes are assigned to the doming fundamental, the iron atom must move in concert with a significant amount of protein material (or else be more strongly damped below the glass transition). More detailed FCS studies of these very interesting low-frequency modes will be reported elsewhere.

Acknowledgment. This work was supported by the National Institutes of Health (AM 35090) and by the National Science Foundation (MCB 9904516). We thank J. T. Sage for helpful discussions.

\section{References and Notes}

(1) Chachisvilis, M.; Fidder, H.; Sundstrom, V. Chem. Phys. Lett. 1995, $234,141-150$.

(2) Dantus, M.; Bowman, R. M.; Gruebele, M.; Zewail, A. H. J. Chem. Phys. 1989, 91, 7437-7450.

(3) Mcmorrow, D.; Lotshaw, W. T.; Kenneywallace, G. A. IEEE J. Ouantum Electron. 1988, 24, 443-454.

(4) Polanyi, J. C.; Zewail, A. H. Acc. Chem. Res. 1995, 28, 119-132.

(5) Pugliano, N.; Szarka, A. Z.; Hochstrasser, R. M. J. Chem. Phys. 1996, $104,5062-5079$

(6) Rosker, M. J.; Wise, F. W.; Tang, C. L. Phys. Rev. Lett. 1986, 57, $321-324$

(7) Scherer, N. F.; Jonas, D. M.; Fleming, G. R. J. Chem. Phys. 1993, 99, $153-168$

(8) Vos, M. H.; Rappaport, F.; Lambry, J. C.; Breton, J.; Martin, J. L. Nature 1993, 363, 320-325.

(9) Wang, Q.; Schoenlein, R. W.; Peteanu, L. A.; Mathies, R. A.; Shank, C. V. Science 1994, 266, 422-424.

(10) Yan, Y. J.; Fried, L. E.; Mukamel, S. J. Phys. Chem. 1989, 93, $8149-8162$.

(11) Zhu, L. Y.; Sage, J. T.; Champion, P. M. Science 1994, 266, 629632.

(12) Ziegler, L. D.; Fan, R.; Desrosiers, A. E.; Scherer, N. F. J. Chem. Phys. 1994, 100, 1823-1839.

(13) Zhu, L.; Li, P.; Huang, M.; Sage, J. T.; Champion, P. M. Phys. Rev. Lett. 1994, 72, 301-304.

(14) Zhu, L. Y.; Wang, W.; Sage, J. T.; Champion, P. M. J. Raman Spectrosc. 1995, 26, 527-534.

(15) Zhu, L. Y.; Zhong, G.; Unno, M.; Sligar, S. G.; Champion, P. M. Biospectroscopy 1996, 2, 301-309.

(16) Rischel, C.; Spiedel, D.; Ridge, J. P.; Jones, M. R.; Breton, J.; Lambry, J. C.; Martin, J. L.; Vos, M. H. Proc. Natl. Acad. Sci. U.S.A. 1998 95, 12306-12311.

(17) Banin, U.; Bartana, A.; Ruhman, S.; Kosloff, R. J. Chem. Phys 1994, 101, 8461-8481.

(18) Chudoba, C.; Lutgen, S.; Jentzsch, T.; Riedle, E.; Woerner, M.; Elsaesser, T. Chem. Phys. Lett. 1995, 240, 35-41.

(19) Dhar, L.; Rogers, J. A.; Nelson, K. A. Chemical Reviews 1994, 94, 157-193.

(20) Kumble, R.; Palese, S.; Visschers, R. W.; Dutton, P. L.; Hochstrasser, R. M. Chem. Phys. Lett. 1996, 261, 396-404.

(21) Lenderink, E.; Duppen, K.; Wiersma, D. A. J. Phys. Chem. 1995, 99, 8972-8977.

(22) Schoenlein, R. W.; Peteanu, L. A.; Wang, Q.; Mathies, R. A.; Shank, C. V. J. Phys. Chem. 1993, 97, 12087-12092.

(23) Wynne, K.; Galli, C.; Hochstrasser, R. M. Chem. Phys. Lett. 1992, $193,17-22$.

(24) Zhu, L. Y.; Widom, A.; Champion, P. M. J. Chem. Phys. 1997, $107,2859-2871$

(25) Champion, P. M.; Rosca, F.; Wang, W.; Kumar, A. T. N.; Christian, J.; Demidov, A. Proc. SPIE in "Laser Techniques for Condensed-Phase and Biological Systems" 1998, 3273, 80-89.

(26) Janes, S. M.; Dalickas, G. A.; Eaton, W. A.; Hochstrasser, R. M. Biophys. J. 1988, 54, 545-549.

(27) Agmon, N.; Doster, W.; Post, F. Biophys. J. 1994, 66, 1612-1622.

(28) Ansari, A.; Jones, C. M.; Henry, E. R.; Hofrichter, J.; Eaton, W. A. Biophys. J. 1993, 64, 852-868.

(29) Jackson, T. A.; Lim, M.; Anfinrud, P. A. Chem. Phys. 1994, 180 $131-140$.

(30) Kaminaka, S.; Ogura, T.; Kitagawa, T. J. Am. Chem. Soc. 1990, $112,23-27$

(31) Lambright, D. G.; Balasubramanian, S.; Boxer, S. G. Chem. Phys. 1991, 158, 249-260.

(32) Nakabayashi, T.; Okamoto, H.; Tasumi, M. J. Phys. Chem. A 1997, $101,3494-3500$.

(33) Tian, W. D.; Sage, J. T.; Srajer, V.; Champion, P. M. Phys. Rev. Lett. 1992, 68, 408-411.

(34) Srajer, V.; Champion, P. M. Biochemistry 1991, 30, 7390-7402.

(35) Sage, J. T.; Schomacker, K. T.; Champion, P. M. J. Phys. Chem. 1995, 99, 3394-3405.

(36) Srajer, V.; Reinisch, L.; Champion, P. M. J. Am. Chem. Soc. 1988 $110,6656-6670$. 
(37) Champion, P. M. J. Raman Spectrosc. 1992, 23, 557-567.

(38) Austin, R. H.; Beeson, K.; Eisenstein, L.; Frauenfelder, H.; Gunsalus, I. C. Biochemistry 1975, 14, 5355-5373.

(39) Walda, K. N.; Liu, X. Y.; Sharma, V. S.; Magde, D. Biochemistry 1994, 33, 2198-2209.

(40) Miers, J. B.; Postlewaite, J. C.; Cowen, B. R.; Roemig, G. R.; Lee,

I. Y. S.; Dlott, D. D. J. Chem. Phys. 1991, 94, 1825-1836.

(41) Ansari, A.; Jones, C. M.; Henry, E. R.; Hofrichter, J.; Eaton, W. A. Science 1992, 256, 1796-1798.

(42) Henry, E. R.; Eaton, W. A.; Hochstrasser, R. M. Proc. Natl. Acad. Sci. U.S.A. 1986, 83, 8982-8986.

(43) Gibson, Q. H.; Olson, J. S.; Mckinnie, R. E.; Rohlfs, R. J. J. Biol. Chem. 1986, 261, 228-239.

(44) Kitagawa T. Biological Applications of Raman Spectroscopy; Wiley and Sons: New York, 1988; p 97.

(45) Constantine, S.; Zhou, Y.; Morais, J.; Ziegler, L. D. J. Phys. Chem. A 1997, 101, 5456-5462.

(46) Yang, T. S.; Chang, M. S.; Chang, R.; Hayashi, M.; Lin, S. H.; Vohringer, P.; Dietz, W.; Scherer, N. F. J. Chem. Phys. 1999, 110, 1207012081 .

(47) Franzen, S.; Lambry, J. C.; Bohn, B.; Poyart, C.; Martin, J. L. Nature Struct. Biol. 1994, 1, 230-233.

(48) Perutz, M. Proc. R. Soc. London B 1980, 135, 208.

(49) Deak, J.; Chin, H. L.; Lewis, C. M.; Miller, R. J. D. J. Phys. Chem.

$B$ 1998, 102, 6621-6634.

(50) Miller, R. J. D. Acc. Chem. Res. 1994, 27, 145-150.

(51) Richard, L.; Genberg, L.; Deak, J.; Chiu, H. L.; Miller, R. J. D. Biochemistry 1992, 31, 10703-10715.

(52) Champion, P. M.; Perreault, G. J. J. Chem. Phys. 1981, 75, 490491.

(53) Champion, P. M.; Lange, R. J. Chem. Phys. 1980, 73, 5947-5957.

(54) Adar, F.; Gouterman, M.; Aronowitz, S. J. Phys. Chem. 1976, 80 2184.

(55) Friedman, J. M.; Rousseau, D. L.; Adar, F. Proc. Natl. Acad. Sci. U.S.A. 1977, 74, 2607-2611.
(56) Friedman, J. M.; Rousseau, D. L. Chem. Phys. Lett. 1978, 55, 488492.

(57) Hochstrasser R. M. Probes of Structure and Function of Macromolecules and Membranes; Academic Press: New York, 1971; p 57.

(58) Petrich, J. W.; Martin, J. L. Chem. Phys. 1989, 131, 31-47.

(59) Cornelius, P. A.; Steele, A. W.; Chernoff, D. A.; Hochstrasser, R. M. Proc. Natl. Acad. Sci. U.S.A. 1981, 78, 7526-7529.

(60) Mizutani, Y.; Kitagawa, T. Science 1997, 278, 443-446. 3227.

(62) Ye et al., to be published.

(63) Scheidt, W. R.; Piciulo, P. J. Am. Chem. Soc. 1976, 98, 1913.

(64) Phillips, S. E. V.; Schoenborn, B. P. Nature 1981, 292, 81-82.

(65) Brucker, E. A.; Olson, J. S.; Ikedasaito, M.; Phillips, G. N. Proteins 1998, 30, 352-356.

(66) Miller, L. M.; Pedraza, A. J.; Chance, M. R. Biochemistry 1997, $36,12199-12207$.

(67) Sage, J. T.; Schomacker, K. T.; Champion, P. M. J. Phys. Chem. 1995, 99, 3394-3405.

(68) Findsen, E. W.; Scott, T. W.; Chance, M. R.; Friedman, J. M.; Ondrias, M. R. J. Am. Chem. Soc. 1985, 107, 3355-3357.

(69) Rosca et al., to be published.

(70) Stavrov, S. S. Biophys. J. 1993, 65, 1942-1950.

(71) Kumar et al., manuscript in preparation.

(72) Jonas, D. M.; Bradforth, S. E.; Passino, S. A.; Fleming, G. R. J. Phys. Chem. 1995, 99, 2594-2608.

(73) Smith, T. J.; Cina, J. A. J. Chem. Phys. 1996, 104, 1272-1292.

(74) Liebl, U.; Lipowski, G.; Negrerie, M.; Lambry, J. C.; Martin, J. L.; Vos, M. H. Nature 1999, 401, 181-184.

(75) Kozlowski, P. M.; Spiro, T. G.; Berces, A.; Zgierski, M. Z. J. Phys. Chem. B 1998, 102, 2603-2608.

(76) Spiro, T. G.; Kozlowski, P. M.; Zgierski, M. Z. J. Raman Spectrosc. 1998, 29, 869-879.

(77) Sage, J. T.; et al., to be published. 